\title{
Electronic structure of $\mathrm{Sn} / \mathrm{Si}(111) \sqrt{3} \times \sqrt{3}$ : Indications of a low-temperature phase
}

\author{
R. I. G. Uhrberg, ${ }^{* 1}$ H. M. Zhang, ${ }^{1}$ T. Balasubramanian, ${ }^{2}$ S. T. Jemander, ${ }^{1}$ N. Lin, ${ }^{1}$ and G. V. Hansson ${ }^{1}$ \\ ${ }^{1}$ Department of Physics and Measurement Technology, Linköping University, S-581 83 Linköping, Sweden \\ ${ }^{2}$ Maxlab, Lund University, Box 118, S-221 00 Lund, Sweden
}

(Received 12 April 2000)

\begin{abstract}
The $\mathrm{Sn} / \mathrm{Si}(111) \sqrt{3} \times \sqrt{3}$ surface has been studied by photoelectron spectroscopy, low-energy electron diffraction (LEED), and scanning tunneling microscopy. Unlike $\mathrm{Sn} / \mathrm{Ge}(111)$, the $\mathrm{Sn} / \mathrm{Si}(111)$ surface shows a $\sqrt{3} \times \sqrt{3}$ LEED pattern at low temperature also $(70 \mathrm{~K})$. The electronic structure, however, is inconsistent with a pure $\sqrt{3} \times \sqrt{3}$ phase. Sn $4 d$ spectra exhibit two major components and the valence band shows two surface bands. These features have been associated with the low-temperature $3 \times 3$ phase in the case of $\mathrm{Sn} / \mathrm{Ge}(111)$. The similarity in the electronic structure points to stabilization of a low-temperature phase for $\mathrm{Sn} / \mathrm{Si}(111)$ also, but at a significantly lower temperature $(<70 \mathrm{~K})$.
\end{abstract}

Phase transitions in low-dimensional systems have recently attracted a lot of experimental and theoretical interest. A striking example is the transition that occurs on the ${ }^{1} \mathrm{~Pb} /$ $\mathrm{Ge}(111)$ and $^{2} \mathrm{Sn} / \mathrm{Ge}(111)$ surfaces. The room-temperature $\sqrt{3} \times \sqrt{3}$ reconstruction, with $\frac{1}{3}$ monolayer of $\mathrm{Pb}$ or $\mathrm{Sn}$ adatoms, changes gradually to a $3 \times 3$ phase when the temperature is lowered. As determined by surface X-ray diffraction, ${ }^{3,4}$ the transition to the $3 \times 3$ phase involves vertical atomic displacements in the adatom layer which give rise to sharp $3 \times 3$ low-energy electron diffraction (LEED) spots. Scanning tunneling microscopy (STM) images of these surfaces show a transition from a $\sqrt{3} \times \sqrt{3}$ to a $3 \times 3$ unit cell, which has been attributed to the formation of a commensurate charge-density wave. ${ }^{1,2}$ Other electronic structure studies, concentrated on the $\mathrm{Sn} / \mathrm{Ge}(111)$ system, have been done by photoelectron spectroscopy. ${ }^{5-7}$ An interesting and rather puzzling result is that the electronic structures of the $\sqrt{3} \times \sqrt{3}$ and $3 \times 3$ surfaces are qualitatively quite similar. The two major $\mathrm{Sn} 4 d$ components and the two surface-state bands that are observed find a natural explanation in a $3 \times 3$ surface phase but are not directly accounted for in a $\sqrt{3} \times \sqrt{3}$ periodicity.

Although the $\mathrm{Pb} / \mathrm{Si}(111)$ and $\mathrm{Sn} / \mathrm{Si}(111)$ systems can be expected to behave in a similar way to their $\mathrm{Ge}(111)$ counterparts, they have been much less studied. The fact that there is no report of a $\sqrt{3} \times \sqrt{3}$ to $3 \times 3$ transition on the $\mathrm{Sn} / \mathrm{Si}(111)$ surface seems to be reflected in the lower number of publications for this system. It is known, however, that the Sn $4 d$ core level of the $\operatorname{Sn} / \operatorname{Si}(111) \sqrt{3} \times \sqrt{3}$ surface shows an unexpected second component. ${ }^{8}$ Inspired by this situation, we have used several techniques to address the interesting atomic and electronic structure of $\mathrm{Sn} / \mathrm{Si}(111)$.

The $\mathrm{Sn} / \mathrm{Si}(111) \sqrt{3} \times \sqrt{3}$ surface has been studied using photoelectron spectroscopy, LEED, and STM. Various Sn coverages were investigated in order to find the optimum preparation of the $\sqrt{3} \times \sqrt{3}$ surface. The use of roomtemperature STM allowed us to check the quality of the surfaces and to characterize the different types of defects that are present. In contrast to the $\mathrm{Sn} / \mathrm{Ge}(111)$ system, we do not observe any transition to a $3 \times 3$ phase in LEED at temperatures down to $70 \mathrm{~K}$, which was the lowest temperature in this study. Despite this difference we find that both the Sn $4 d$ core-level and valence-band spectra show the features that have been attributed to the $3 \times 3$ phase for the $\mathrm{Sn} / \mathrm{Ge}(111)$ system..$^{5-7}$ It is quite intriguing that a second $\mathrm{Sn} 4 d$ component and a second dangling-bond band show up in the photoemission experiment for the $\mathrm{Sn} / \mathrm{Si}(111)$ system also even though there are no signs of a $3 \times 3$ phase. There is, however, an interesting difference in the core-level results. In contrast to the $\mathrm{Sn} / \mathrm{Ge}(111)$ surface the smaller $\mathrm{Sn} 4 d$ component appears at the lower-binding-energy side of the larger component. This difference, which may be related to the different low-temperature behavior of the $\mathrm{Sn} / \mathrm{Si}(111)$ surface, can be qualitatively explained by the two different types of $3 \times 3$ disturbance that can be induced by substitutional defects. $^{9,10}$

Photoemission and LEED studies were performed at beam line 33 at the MAX-I synchrotron radiation facility in Lund, Sweden. The angle-resolved valence-band spectra were obtained with a total energy resolution of $\approx 50 \mathrm{meV}$ and an angular resolution of $\pm 2^{\circ}$. The Sn $4 d$ core-level spectra were measured at an energy resolution of $\approx 90 \mathrm{meV}$ with the same angular resolution. STM and LEED studies were performed in an experimental chamber at Linköping University. ${ }^{11}$ The $\mathrm{Si}(111)$ samples (Sb doped, $3 \Omega \mathrm{cm}$ ) were cleaned and preoxidized before insertion into the vacuum chambers and the chemical oxide was removed in situ by direct-current heating of the sample to either $\approx 930$ or $\approx 1150{ }^{\circ} \mathrm{C}$. Sn was evaporated onto the samples from a filament source calibrated by a quartz crystal monitor. After evaporation, the $\mathrm{Si}(111)$ samples were annealed to $\approx 650^{\circ} \mathrm{C}$ to form ordered surfaces. This annealing temperature has earlier been reported to give $\sqrt{3} \times \sqrt{3}$ surfaces with the lowest defect density (substitutional Si atoms). ${ }^{12}$ The amount of Sn deposited onto the surfaces, $\theta_{\mathrm{Sn}}$, in monolayers (ML) has been used to label the different sample preparations. We do not claim that these numbers correspond exactly to the absolute coverage. An error on the order of $5-10 \%$ is probably a realistic estimate.

Figure 1 shows a set of $\mathrm{Sn} 4 d$ spectra measured at normal emission for initial Sn converages between 0.20 and 0.47 ML. The spectrum for the lowest Sn coverage, which corresponds to the so called "mosaic", phase, shows essentially one spin-orbit-split $4 d$ component. As the coverage is in- 


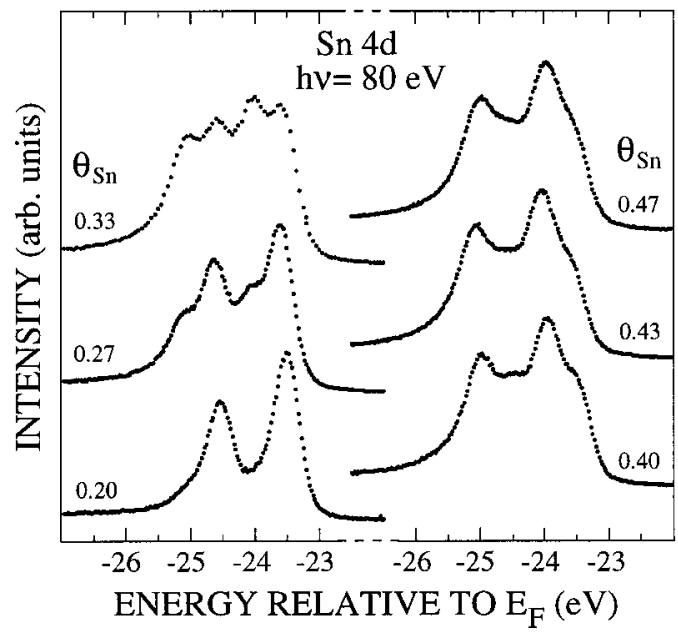

FIG. 1. Sn $4 d$ core-level spectra obtained at room temperature. The amount of Sn evaporated onto the $\mathrm{Si}(111)$ surface is indicated by $\theta_{\text {Sn }}(\mathrm{ML})$. All surfaces were annealed to $650{ }^{\circ} \mathrm{C}$ for $2 \mathrm{~min}$. The spectra are displayed with the same peak height.

creased a second component develops on the left, highbinding-energy, side. The spectra continue to change up to 0.40 ML of Sn while the spectra for this and higher coverages look essentially identical. The different preparations were also investigated by LEED. All surfaces showed a $\sqrt{3}$ $\times \sqrt{3}$ LEED pattern but significant differences were found in the background between the diffraction spots. For initial Sn coverages less than $0.40 \mathrm{ML}$ we observed weak, diffuse streaks between $1 \times 1$ spots. For initial coverages between 0.40 and $0.47 \mathrm{ML}$ the streaks were no longer observed. If the initial Sn coverage was higher than 0.47 ML the LEED patterns showed diffraction from $2 \sqrt{3} \times 2 \sqrt{3}$ areas on the surface. Based on the LEED results we define the optimum preparation of the $\sqrt{3} \times \sqrt{3}$ surface as one that does not show any streaks between the $1 \times 1$ spots or any indication of $2 \sqrt{3} \times 2 \sqrt{3}$ spots. This situation was achieved by depositing 0.40-0.47 ML of $\mathrm{Sn}$ on the $\mathrm{Si}(111) 7 \times 7$ surface followed by annealing at $650{ }^{\circ} \mathrm{C}$ for $2 \mathrm{~min}$. As can be seen from Fig. 1 the $\mathrm{Sn} 4 d$ spectra all look very similar in this coverage range and any of these three spectra should be representative of the $\sqrt{3} \times \sqrt{3}$ surface. These spectra are similar to spectra in the literature. ${ }^{8}$ From the line shape it is quite clear that the $\mathrm{Sn} 4 d$ spectra consist of at least two components, which is in contrast to the single component expected for a simple $\sqrt{3} \times \sqrt{3}$ surface.

As a third way of characterizing the $\operatorname{Sn} / \operatorname{Si}(111) \sqrt{3} \times \sqrt{3}$ surface we have performed STM studies of roomtemperature samples. Five independent sample preparations were investigated, which were all essentially identical to the preparations used for the photoemission study of the optimized $\sqrt{3} \times \sqrt{3}$ surface. Figure 2 shows a typical filled-state STM image of such a surface. A number of typical defects can be identified in this image. The most striking features are the bright hexagons with a dark center $(S)$. In empty-state images the centers appear as gray features indicating that the atomic position is not vacant. In accordance with earlier STM studies, we attribute the bright hexagons to $\mathrm{Sn}$ atoms surrounding a Si substitutional atom. ${ }^{2,13,14}$ The average concentration of the $\mathrm{Si}$ atoms for the five different preparations was $\approx 1 \%$ of the adatoms with a very small spread. A second

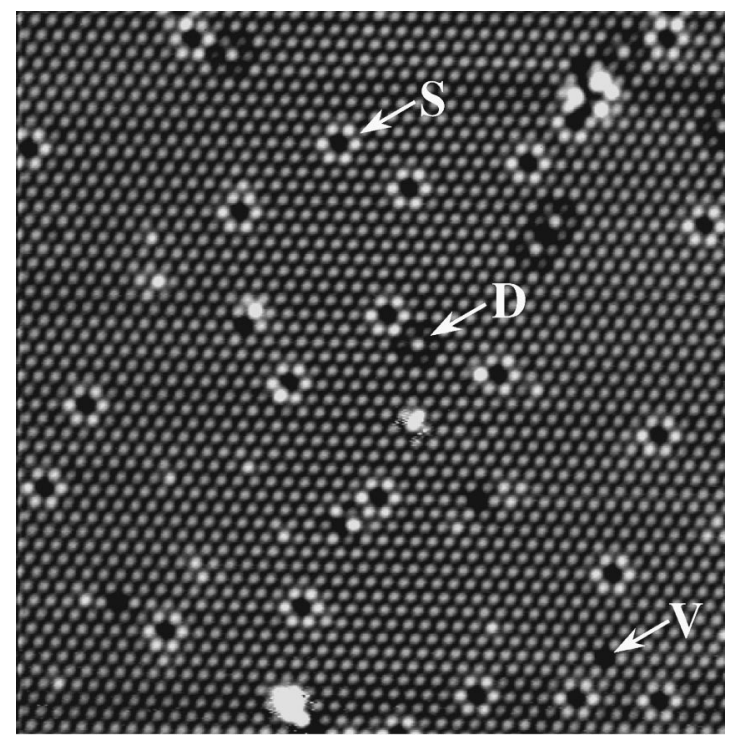

FIG. 2. Filled-state STM image $\left(282 \times 294 \AA^{2}\right)$ of the $\sqrt{3} \times \sqrt{3}$ surface obtained at room temperature $(1.0 \mathrm{~V}$ tip bias, $300 \mathrm{pA})$. The three different types of defects, $S, V$, and $D$ are discussed in the text.

type of defect appears as a dark atomic position surrounded by six atoms with no specific contrast $(V)$. This defect appears dark also in empty-state images and is interpreted as a real vacancy as in other studies. ${ }^{2,13,14}$ The average concentration of vacancies $(\approx 0.2 \%)$ is significantly smaller than the concentration of $\mathrm{Si}$ substitutional defects. A third type of defect shows up as a bright spot surrounded by six atoms that are slightly darker than the normal $\sqrt{3} \times \sqrt{3}$ Sn atoms $(D)$. In reverse-bias images the bright center spot appears dark, similar to a vacancy. This behavior is what one can expect from group-V substitutional atoms. Since the $\mathrm{Si}$ substrates were doped with $\mathrm{Sb}$ atoms we interpret this feature as due to substitutional doping atoms. The average concentration of these defects is $\approx 0.2 \%$. From the STM studies we conclude that the preparation method used for the LEED and photoemission studies results in a surface with a small number of defects. The total defect concentration is less than $1.5 \%$ with the $\mathrm{Si}$ substitutional atom as the most common one $(\approx 1 \%)$.

Because of the strong interest in the $\sqrt{3} \times \sqrt{3}$ to $3 \times 3$ transition observed in the $\mathrm{Sn} / \mathrm{Ge}(111)$ system, it is quite interesting to study the $\operatorname{Sn} / \operatorname{Si}(111) \sqrt{3} \times \sqrt{3}$ surface also at low temperature. Figure 3 shows a LEED pattern obtained at a sample temperature of $70 \mathrm{~K}$ from a surface with an initial $\mathrm{Sn}$ coverage of $0.40 \mathrm{ML}$. This LEED image is overexposed to allow a detailed study of the weak background intensity. In contrast to the $\mathrm{Sn} / \mathrm{Ge}(111)$ case we are not able to identify any $3 \times 3$ diffraction spots despite the enhanced background sensitivity. We therefore conclude that the $\mathrm{Sn} / \mathrm{Si}(111)$ surface does not undergo the $\sqrt{3} \times \sqrt{3}$ to $3 \times 3$ structural transition as observed for $\mathrm{Sn} / \mathrm{Ge}(111)$, at least not for temperatures down to $70 \mathrm{~K}$.

Photoemission, however, provides a quite different view of the $\sqrt{3} \times \sqrt{3}$ surface. Figure 4 shows a Sn $4 d$ core-level spectrum recorded at $70 \mathrm{~K}$ from the surface corresponding to the LEED image in Fig. 3. The decomposition of the spectrum shows two major components $C_{1}$ and $C_{2}$ and a minor component $C_{3}$. The relative intensities of $C_{1}, C_{2}$, and $C_{3}$ 


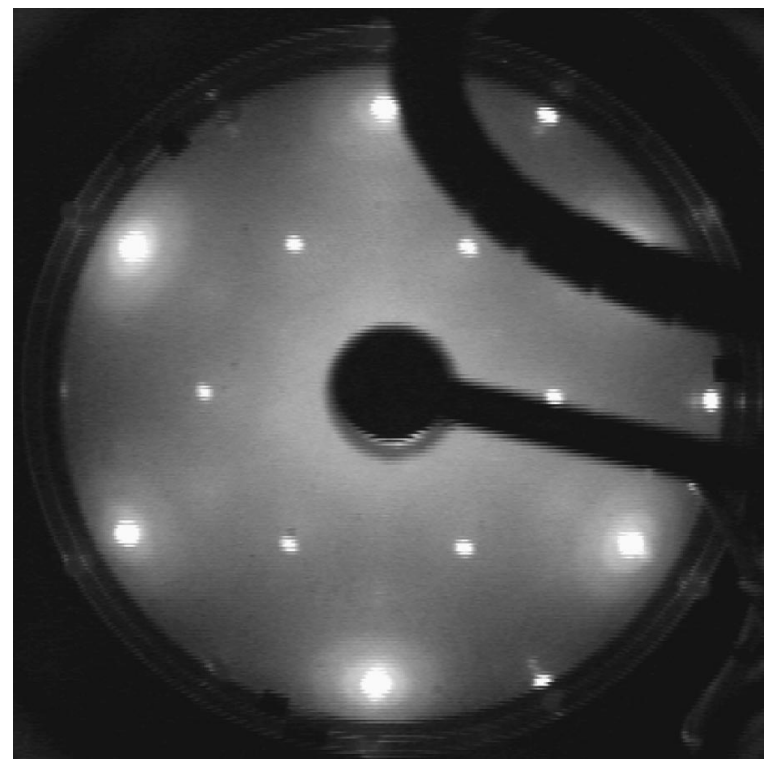

FIG. 3. LEED image obtained at $70 \mathrm{~K}$ showing only $\sqrt{3} \times \sqrt{3}$ diffraction (37 eV electron energy).

are 30,63 , and $7 \%$, respectively. Although the LEED pattern shows no sign of any $3 \times 3$ periodicity, the $\operatorname{Sn} 4 d$ spectrum clearly shows two major components. The intensity ratio $63 / 30=2.1$ is close to the expected ratio of 2 for a $3 \times 3$ reconstruction. There is, however, a significant difference in the binding energies of the $C_{1}$ and $C_{2}$ components compared to the $\mathrm{Sn} / \mathrm{Ge}(111)$ system. $^{5-7}$ The smaller component $C_{1}$ is located to the right of the larger component $C_{2}$, which is the opposite of the $\mathrm{Sn} / \mathrm{Ge}(111)$ case. $^{8}$ At room temperature the Sn $4 d$ core-level spectra look very similar except for a general broadening (see Fig. 1) and they decompose into the same three components.

The $\mathrm{Sn}$ atoms forming the bright hexagons around the $\mathrm{Si}$ defects are obviously different from the other $\mathrm{Sn}$ atoms and one might guess that they give rise to the $C_{1}$ component. A

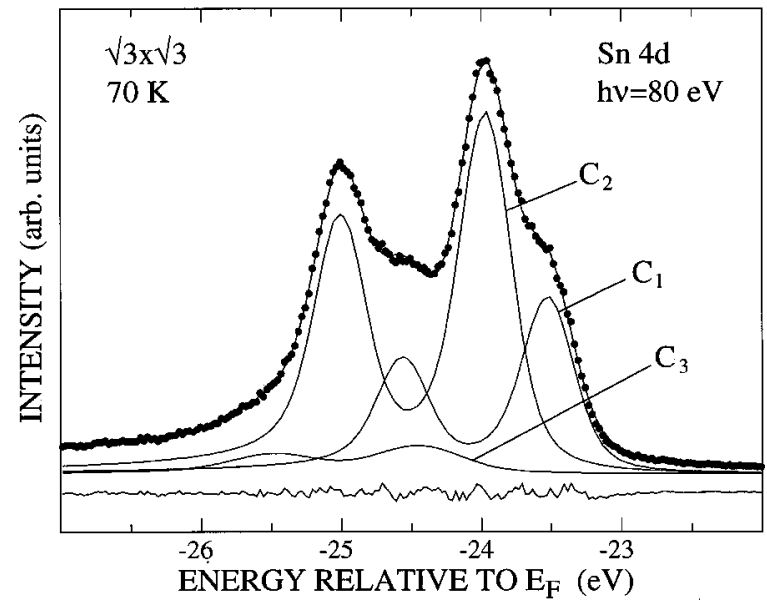

FIG. 4. Sn $4 d$ core-level spectrum recorded at $70 \mathrm{~K}$ from the surface corresponding to the LEED image in Fig. 3. The spectrum decomposes into three components $C_{1}, C_{2}$, and $C_{3}$. The fitting parameters were spin-orbit split 1.04 , branching ratio $0.65 \pm 0.03$, Lorentzian width 0.175 , singularity index $\alpha=0.06$. The Gaussian width of $C_{1}$ and $C_{2}$ was $0.33 \mathrm{eV}$ and $0.63 \mathrm{eV}$ for $C_{3} . C_{2}-C_{1}=$ $-0.45 \mathrm{eV}$ and $C_{2}-C_{3}=0.47 \mathrm{eV}$.

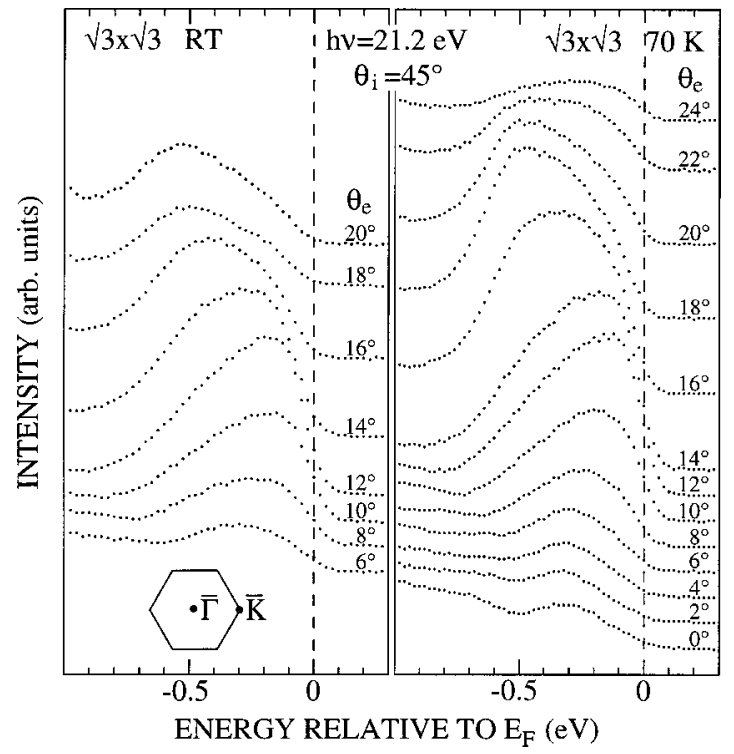

FIG. 5. Angle-resolved valence-band spectra from the $\mathrm{Sn} /$ $\mathrm{Si}(111) \sqrt{3} \times \sqrt{3}$ surface recorded at RT and at $70 \mathrm{~K}$. The surfacestate emission is shown at different emission angles $\theta_{e}$ in the $\bar{\Gamma}-\bar{K}$ direction of the $\sqrt{3} \times \sqrt{3}$ surface Brillouin zone. The $\bar{K}$ point corresponds to $\theta_{e}=18^{\circ}$.

quick analysis shows, however, that the intensity of $C_{1}$ is far too high for such an identification. The number of $\mathrm{Sn}$ atoms in the bright hexagons constitutes less than $6 \%$ of the total number of $\mathrm{Sn}$ atoms, which should be compared to the intensity of $C_{1}$ of $30 \%$. With this big discrepancy we will have to look for an explanation of $C_{1}$ that involves the $\mathrm{Sn}$ atoms that appear to be ordered in a simple $\sqrt{3} \times \sqrt{3}$ periodicity. The small concentration of hexagon atoms instead suggests an identification with the small $C_{3}$ component with a relative intensity of $\approx 7 \%$.

Angle-resolved valence-band photoemission provides another vital piece of information about the $\sqrt{3} \times \sqrt{3}$ and $3 \times 3$ reconstructions. For $\mathrm{Sn} / \mathrm{Ge}(111)$ it has been shown that both the $\sqrt{3} \times \sqrt{3}$ and $3 \times 3$ surfaces exhibit two dispersive surface-state bands. ${ }^{6,7}$ The two bands are not resolved at room temperature (RT) and they give rise to an asymmetric peak in the angle-resolved spectra. At low temperature $(\approx 100 \mathrm{~K})$ the two peaks are separated and the dispersion can be easily determined. The existence of two bands at RT for $\mathrm{Sn} / \mathrm{Ge}(111)$ has been used as evidence for the presence of the $3 \times 3$ periodicity above what is regarded to be the $\sqrt{3} \times \sqrt{3}$ to $3 \times 3$ transition temperature. ${ }^{6,7}$ Figure 5 shows valence-band spectra obtained along the $\bar{\Gamma}-\bar{K}$ line of the $\sqrt{3} \times \sqrt{3}$ surface Brillouin zone for the $\mathrm{Sn} / \mathrm{Si}(111)$ surface at both RT and 70 $\mathrm{K}$. These spectra look rather similar to the corresponding spectra of $\mathrm{Sn} / \mathrm{Ge}(111),{ }^{6,7}$ except for one difference. The two surface bands, which are not resolved at RT, become clearly resolved for the low-temperature $3 \times 3$ phase of $\mathrm{Sn} / \mathrm{Ge}(111)$. For $\mathrm{Sn} / \mathrm{Si}(111)$ we do not observe any phase transition and the appearance of the spectra is more or less the same throughout the temperature interval. However, the main result from the valence-band data is quite clear. From the asymmetric peak shape, throughout the angle series, we can conclude that the $\mathrm{Sn} / \mathrm{Si}(111)$ surface also shows two surfacestate structures despite the fact that no $3 \times 3$ periodicity could be detected by LEED or STM. The surface band struc- 
ture is metallic, both at RT and at $70 \mathrm{~K}$, as shown by the clear Fermi-level crossing at $\theta_{e}=10^{\circ}$. At smaller emission angles a surface-state structure remains well below the Fermi level, which can be attributed to a second fully occupied surface-state band in similarity with the $\mathrm{Sn} / \mathrm{Ge}(111)$ system.

For the $\mathrm{Sn} / \mathrm{Ge}(111)$ system the strong second $\mathrm{Sn} 4 d$ component can be qualitatively explained by a reconstruction that is stabilized by the presence of Ge substitutional defects. These defects seem to induce a local $3 \times 3$ periodicity at room temperature, ${ }^{2,14}$ which extends over larger areas with decreasing temperature as observed by STM. Recently, new and more detailed information about the characteristics of $\mathrm{Ge}$ defects on $\mathrm{Sn} / \mathrm{Ge}(111),{ }^{9}$ and $\mathrm{Si}$ defects on $\mathrm{Sn} / \mathrm{Si}(111),{ }^{10}$ was published. It was reported that Ge defects can induce two types of $3 \times 3$ pattern on the surface. The $3 \times 3$ periodicity that is normally reported for the $\mathrm{Sn} / \mathrm{Ge}(111)$ surface $^{2}$ gives rise to one bright and two dark Sn atoms per $3 \times 3$ unit cell in filled-state STM images (the bright atoms form a hexagonal pattern). In Ref. 9 this pattern is explained by the superposition of the influence from Ge defects located on different 3 $\times 3$ sublattices. If the Ge defects are isolated or located on the same sublattice they instead give rise to a different type of disturbance of the neighboring Sn atoms (the bright atoms form a honeycomb pattern in this case). In filled-state STM images the $3 \times 3$ unit cell of the honeycomb type contain two bright and one dark $\mathrm{Sn}$ atom, i.e., a reversal of the hexagonal pattern. A similar honeycomb-type disturbance has been reported in a STM study of single $\mathrm{Si}$ defects on $\mathrm{Sn} / \mathrm{Si}(111)$ at $120 \mathrm{~K}$. Based on the significantly smaller concentration of substitutional defects for $\mathrm{Sn} / \mathrm{Si}(111)(\approx 1 \%)$ compared to $\mathrm{Sn} /$ $\mathrm{Ge}(111)[3 \pm 1 \%$ (Ref. 9)] and the shorter decay length of the disturbance caused by a defect $[11 \AA$ for $\operatorname{Sn} / \mathrm{Si}(111)$ at $120 \mathrm{~K},{ }^{10}$ compared to $50 \AA$ for $\mathrm{Sn} / \mathrm{Ge}(111$ ) (Ref. 9)], one can expect the $\mathrm{Si}$ defects on the $\mathrm{Sn} / \mathrm{Si}(111)$ surface to be essentially isolated without any significant interference between them. The dominating disturbance caused by the Si defects can therefore be expected to be of the honeycomb type, while the hexagonal $3 \times 3$ pattern is dominant for $\mathrm{Sn} / \mathrm{Ge}(111) .{ }^{9}$ In the case of $\mathrm{Sn} / \mathrm{Ge}(111)$ the smaller of the two $4 d$ components, which is associated with the bright $\mathrm{Sn}$ atoms, has a higher binding energy than the larger component, which is due to the two dark Sn atoms. ${ }^{15}$ In the honeycomb reconstruction there are instead two bright atoms and one dark atom and one therefore expects to find the larger component at the high-binding-energy side in that case. Since this is exactly what we observe (see Fig. 4) it is tempting to propose that the $\mathrm{Sn} / \mathrm{Si}(111)$ surface can be described by the honeycomb-type $3 \times 3$ reconstruction. However, the lack of $3 \times 3$ diffraction spots at $70 \mathrm{~K}$ seems to be in contradiction with such an explanation. This type of conflicting results obtained by photoelectron spectroscopy and LEED was discussed earlier for $\mathrm{Sn} / \mathrm{Ge}(111){ }^{6,7}$ The characteristic features of the $\mathrm{Sn} 4 d$ and valence-band spectra associated with the $3 \times 3$ periodicity are present at RT although no diffraction spots from this phase can be observed by LEED.

This apparent discrepancy between the LEED and the photoemission results can be explained in terms of thermally induced fluctuations similar to the situation for the $\operatorname{Si}(100) c(4 \times 2)$ to $2 \times 1$ transition. ${ }^{6,7}$ The dynamical fluctuations of the $\mathrm{Sn}$ atom positions have been treated theoretically for $\mathrm{Sn} / \mathrm{Ge}(111)$ in Ref. 7, where it was concluded that thermal fluctuations could lead to a situation where the Sn atoms are located instantaneously in either of the two vertical positions of the $3 \times 3$ phase. This could explain why a "time averaging', technique like STM shows a $\sqrt{3} \times \sqrt{3}$ periodicity near RT, and why the $3 \times 3$ diffraction gradually disappears with increasing temperature. Photoelectron spectroscopy, which works on a much shorter time scale, would, however, still show the essential features of the $3 \times 3$ reconstruction. One merit of the "dynamical fluctuation model" is that it can explain the strong intensity of the extra $\mathrm{Sn} 4 d$ component at RT and the small difference between low-temperature and RT spectra. The results of a recent electron standingwave measurement of the $\mathrm{Sn}$ adatom heights on the $\mathrm{Sn} / \mathrm{Si}(111) \sqrt{3} \times \sqrt{3}$ surface are consistent with dynamical fluctuations. ${ }^{16}$

In conclusion, we find that the $\mathrm{Sn} 4 d$ and valence-band spectra of the $\operatorname{Sn} / \operatorname{Si}(111) \sqrt{3} \times \sqrt{3}$ surface show the characteristic features that have been attributed to a $3 \times 3$ periodicity in the case of $\mathrm{Sn} / \mathrm{Ge}(111)$. These results indicate that the $\mathrm{Sn} / \mathrm{Si}(111)$ system may undergo a similar phase transition but at a significantly lower temperature $(<70 \mathrm{~K})$. Based on the strong intensity of both the $C_{1}$ and $C_{2}$ components, we assign them to inequivalent $\mathrm{Sn}$ atoms on the seemingly $\sqrt{3} \times \sqrt{3}$ reconstructed areas. An intriguing difference in the $\mathrm{Sn} 4 d$ spectra of the $\mathrm{Sn} / \mathrm{Si}(111)$ and $\mathrm{Sn} / \mathrm{Ge}(111)$ systems was pointed out. The smaller component $C_{1}$ appears at the low binding-energy side of the large component $C_{2}$ in $\mathrm{Sn} /$ $\mathrm{Si}(111)$. This is opposite to what is observed on the $\mathrm{Sn} /$ Ge(111) surface. This difference can be understood if the $\mathrm{Sn} / \mathrm{Si}(111)$ surface is described by the honeycomb-type reconstruction instead of the hexagonal reconstruction that is valid for $\mathrm{Sn} / \mathrm{Ge}(111)$.

Support from the Maxlab staff is gratefully acknowledged. This work was supported by the Swedish Natural Science Research Council.
*Corresponding author. FAX: (+46) 13137568.

Email: rub@ifm.liu.se

${ }^{1}$ J. M. Carpinelli, H. H. Weitering, E. W. Plummer, and R. Stumpf, Nature (London) 381, 398 (1996).

${ }^{2}$ J. M. Carpinelli, H. H. Weitering, M. Bartkowiak, R. Stumpf, and E. W. Plummer, Phys. Rev. Lett. 79, 2859 (1997).

${ }^{3}$ A. Mascaraque, J. Avila, J. Alvarez, M. C. Asensio, S. Ferrer, and E. G. Michel, Phys. Rev. Lett. 82, 2524 (1999).

${ }^{4}$ O. Bunk, J. H. Zeysing, G. Falkenberg, R. L. Johnson, M. Nielsen, M. M. Nielsen, and R. Feidenhans'1, Phys. Rev. Lett. 83, 2226 (1999).
${ }^{5}$ G. Le Lay, V. Yu. Aristov, O. Boström, J. M. Layet, M. C. Asensio, J. Avila, Y. Huttel, and A. Cricenti, Appl. Surf. Sci. 123/124, 440 (1998).

${ }^{6}$ R. I. G. Uhrberg and T. Balasubramanian, Phys. Rev. Lett. 81, 2108 (1998).

${ }^{7}$ J. Avila, A. Mascaraque, E. G. Michel, M. C. Asensio, G. Le Lay, J. Ortega, R. Pérez, and F. Flores, Phys. Rev. Lett. 82, 442 (1999).

${ }^{8}$ M. Göthelid, M. Björkqvist, T. M. Grehk, G. Le Lay, and U. O. Karlsson, Phys. Rev. B 52, 14352 (1995), and references therein. 
${ }^{9}$ A. V. Melechko, J. Braun, H. H. Weitering, and E. W. Plummer, Phys. Rev. B 61, 2235 (2000).

${ }^{10}$ L. Ottaviano, M. Crivellari, L. Lozzi, and S. Santucci, Surf. Sci. 445, L41 (2000).

${ }^{11}$ F. Owman and P. Mårtensson, Surf. Sci. 324, 211 (1995).

${ }^{12}$ C. Törnevik, M. Göthelid, M. Hammar, U. O. Karlssson, N. G. Nilsson, S. A. Flodström, C. Wigren, and M. Östling, Surf. Sci. 314, 179 (1994).

${ }^{13}$ H. H. Weitering, J. M. Carpinelli, A. V. Melechko, J. Zhang, M. Bartkowiak, and E. W. Plummer, Science 285, 2107 (1999).
${ }^{14}$ A. V. Melechko, J. Braun, H. H. Weitering, and E. W. Plummer, Phys. Rev. Lett. 83, 999 (1999).

${ }^{15}$ The Sn $4 d$ core-level spectra and the decompositions presented in Refs. 5-7 were recently questioned by T. E. Kidd et al. [Phys. Rev. Lett. 83, 2789 (1999)]. We have performed new LEED and PES studies which unambiguously confirm that the Sn $4 d$ spectra presented by Refs. 5-7 are representative of the $3 \times 3$ and $\sqrt{3} \times \sqrt{3}$ surfaces of $\mathrm{Sn} / \mathrm{Ge}(111)$ [R. I. G. Uhrberg et al., Phys. Rev. Lett. 85, 1036 (2000)].

${ }^{16}$ T. Yamanaka and S. Ino, Phys. Rev. B 61, R5074 (2000). 